Tropical Journal of Pharmaceutical Research January 2014; 13 (1): 31-39

ISSN: $1596-5996$ (print); 1596-9827 (electronic)

(C) Pharmacotherapy Group, Faculty of Pharmacy, University of Benin, Benin City, 300001 Nigeria.

All rights reserved.

Available online at http://www.tjpr.org

Original Research Article

http://dx.doi.org/10.4314/tjpr.v13i1.5

\title{
Olea europaea Linn (Oleaceae) Fruit Pulp Exhibits Hypocholesterolemic and Hepatoprotective Effects via Regulation of Peroxisome Proliferation-Activated Receptor Alpha in High-Fat Diet-Fed Rats
}

\author{
Moo-Sung Kim², Sushruta Koppula ${ }^{1}$, Soo-Jin Sung ${ }^{1,3,4}$, Sang-Rin Lee ${ }^{2}$, Yong- \\ Dae Park ${ }^{2}$, Kyung-Ae Lee ${ }^{2}$, Tae-Kyu Park ${ }^{1}$ and Hyun Kang ${ }^{1^{*}}$ \\ ${ }^{1}$ Department of Biotechnology, Research Institute for Biomedical \& Health Science, College of Biomedical and Health Science, \\ Konkuk University, Chungju, 380-701, ${ }^{2}$ R\&D Center, Macrocare Tech, Ltd, Ochang, 363-883, ${ }^{3}$ KuGen Healthcare Institute, \\ Chungju, 380-150, ${ }^{4}$ Nutra MediPharm, Chungju, 380-701, Republic of Korea.
}

*For correspondence: Email: hkang@kku.ac.kr; Tel: 82-43-840-3603; Fax: 82-43-852-3616

\begin{abstract}
Purpose: To investigate the hypocholesterolemic and hepatoprotective effects of Olea europaea Linn. (Oleaceae) fruit pulp (OFP-EA) extract in experimental rats.

Methods: Sprague-Dawley rats were fed with a normal diet, a high-cholesterol diet or high-cholesterol diets supplemented with OFP-EA extract (100 or $300 \mathrm{mg} / \mathrm{kg} / \mathrm{day}$ p.o.) for 28 days. Plasma lipid profile such as total cholesterol (TC), triglycerides (TG), high-density lipoprotein (HDL) and low-density lipoprotein ( $L D L)$ were measured using their respective commercial kits. Gross appearance and lipid content of liver samples were also analyzed. The changes in protein expressions of key enzymes such as hydroxymethylglutaryl-CoA reductase (HMGR), acyl-CoA:cholesterol acyltransferase (ACAT), cytochrome P450 7A1 (CYP7A1) and peroxisome proliferation-activated receptor alpha (PPAR- $\alpha$ ) in rat livers were evaluated using Western blotting.

Results: OFP-EA-extract markedly altered the increased plasma TC, TG, LDL and decreased the HDL induced by a hypercholesterolemic diet with a dose-dependent improvement on both atherogenic index and cardiac risk factor ( $p<0.01$ and $p<0.001$ at 100 and $300 \mathrm{mg} / \mathrm{kg}$ dose, respectively). Lipid deposition in liver was attenuated significantly in hypercholesterolemic animals supplemented with OFPEA-extract ( $p<0.01$ at 100 and $p<0.001$ at $300 \mathrm{mg} / \mathrm{kg}$ dose, respectively). Further mechanistic studies revealed that OFP-EA extract regulated the altered expressional levels of CYP7A1 and PPAR- $\alpha$ in the livers of hypercholesterolemic diet-induced rats.

Conclusion: The hypocholesterolemic and hepatoprotective functions of OFP-EA-extract are probably due to the increase in fatty acids unitization in liver via the up-regulation of PPAR- $\alpha$ level.
\end{abstract}

Keywords: Olea europaea, cholesterol, Hypercholesterolemia, Lipid metabolism, Peroxisome proliferation-activated receptor alpha.

Tropical Journal of Pharmaceutical Research is indexed by Science Citation Index (SciSearch), Scopus, International Pharmaceutical Abstract, Chemical Abstracts, Embase, Index Copernicus, EBSCO, African Index Medicus, JournalSeek, Journal Citation Reports/Science Edition, Directory of Open Access Journals (DOAJ), African Journal Online, Bioline International, Open-J-Gate and Pharmacy Abstracts

\section{INTRODUCTION}

Olea europaea Linn. from the family, Oleaceae, commonly known as olive is among the oldest known cultivated trees in the world with immense medicinal values. The beneficial effects of a Mediterranean diet rich in olive oil and table olives have been widely reported [1,2]. Olive products are rich in natural antioxidants that may inhibit oxidative stress during the development of major diseases such as coronary heart disease 
$[3,4]$, cancer [5] and neurodegenerative diseases [6]. The antioxidant properties of olive products have been attributed to the presence of phenolic compounds [7] which act by scavenging free radicals and chelating metallic ions [1]. After the extraction of oil from the olive, many phenolic compounds remain in the by-products. Olive-mill waste water known as "alpechin" and olive oil cake known as "alperujo" contain a similar phenolic profile to that of the original fruit [8]. Earlier studies reported by our group on olive fruit pulp ethyl acetate (OFP-EA) extract revealed a strong anti-oxidant and anti-neuroinflammatory properties [6]. In the present study we investigated the hypocholesterolemic and hepatoprotective effects OFP-EA extract in normal and hypocholesterolemic-induced experimental rats.

Cardiovascular disease (CVD) is a leading cause of death and its incidence rate is increasing day by day in the developing world. Although the progression of CVD is multifactorial, modern lifestyle with high-cholesterol diet and less physical activity contributes to hypercholesterolemia which is believed to play an important role in the pathogenesis and progression of CVD [9, 10]. In addition, hypercholesterolemia can result in non-alcoholic fatty liver disease (NAFLD), which is a pathological condition of emerging clinical importance and considered as the most common cause of abnormal liver function [11]. NAFLD is characterized by a wide spectrum of liver damage, such as simple steatosis, fibrosis and cryptogenic cirrhosis [11]. The progression of the disease involves increased inflammation and oxidative stress leading to lipid peroxidation [12]. Thus, controlling plasma cholesterol or preventing hypercholesterolemia is an ideal approach to control the development of CVD and NAFLD. Although several drugs are available clinically, researches have focused on the consumption of anti-oxidant rich dietary supplements and functional foods from natural herbs in lowering and/or controlling hypercholesterolemia and NAFLD. [13].

Therefore, we investigated the effects of OFP-EA extract on normal and high fat diet (HFD) fed rats and evaluated the plasma lipid profiles such as triglycerides (TG), total cholesterol (TC), highdensity lipoprotein (HDL) and low-density lipoprotein (LDL)] in the liver. Further, the atherogenic index, cardiac risk factor, total lipid level and the changes in the protein expressions of key enzymes such as hydroxymethylglutarylCoA reductase (HMGR), acyl-CoA:cholesterol acyltransferase (ACAT), cytochrome P450 7A1 (CYP7A1) and peroxisome proliferation-activated receptor alpha (PPAR- $\alpha$ ), responsible for the cholesterol homeostasis were studied in the livers so as to understand its effect on hepatic fatty acid uptake and oxidation.

\section{EXPERIMENTAL}

\section{Chemicals}

Dulbecco's modified Eagle medium (DMEM), fetal bovine serum (FBS), and penicillin were purchased from HyClone Laboratories, Inc. (South Logan, Utah). The kits for analyzing plasma lipid profile were purchased from Asan Biotech. Co. Ltd. (Seoul, Korea). All other reagents are of highest grade commercially available.

\section{Preparation of OFP-EA extract}

The OFP-EA extract was prepared as described in our earlier published work [6] . Briefly, Green olive fruits collected at the end of September to about the middle of November were obtained from the local market, Seoul, South Korea. The collected fruit material was authenticated by Prof Jong-Bo Kim, a taxonomist at Konkuk University, Korea and a voucher specimen (OL-KU2012) has been kept in our laboratory herbarium, Konkuk University, Korea for future reference. To obtain the olive fruit pulp extract, $500 \mathrm{~g}$ of the fruit were ground in a mixer and defatted three times with three volumes of $80 \%$ ethanol. The residue (fruit pulp) was extracted with absolute ethanol $(\mathrm{EtOH})$ at 1:10 ratio (w/v) for $2 \mathrm{~h}$ in a heating mantle at $70-80{ }^{\circ} \mathrm{C}$. The supernatant was filtered and concentrated in a rotatory evaporator at $50{ }^{\circ} \mathrm{C}$. The ethanol extract of olive fruit pulp obtained $(180 \mathrm{~g})$ was re-suspended in water:EtOH $(9: 1, \mathrm{v} / \mathrm{v})$ and partitioned successively with n-hexane, ethyl acetate (EA) and n-butanol to obtain a final yield of 19.4, 52 and $27.27 \%$, respectively. EA extract was dissolved in sterile distilled water and filtered on $0.22 \mu \mathrm{m}$ filter before use.

\section{HPLC finger print analysis of OFP-EA extract}

A Shimadz system equipped with binary pumps, an inline degasser, an autosampler, and a PAD detector was used. UV spectra were recorded between 200 and $450 \mathrm{~nm}$ and the UV trace was measured at $360 \mathrm{~nm}$. A Symmetry C18 analytical column (250' $4.6 \mathrm{~mm}$ i.d.; particle size, $5 \mu \mathrm{m})$ fitted with a Novapack RP C18 guard column (Waters, Millford, MA) were used for the separation. The mobile phase consisted of $0.5 \%$ formic acid in deionized water (A) and $0.5 \%$ formic acid in acetonitrile:methanol (50:50, v/v) (B). The gradient program was as follows: $25 \%$ 
$\mathrm{B}$ to $40 \% \mathrm{~B}$ in $20 \mathrm{~min}, 40 \% \mathrm{~B}$ to $50 \% \mathrm{~B}$ in 5 $\min , 50 \%$ to $100 \% \mathrm{~B}$ in $5 \mathrm{~min}$ followed by $100 \%$ $B$ in $5 \mathrm{~min}$ at a flow rate of $1 \mathrm{~mL} \min -1$. There was a 10-min post-run going back to the starting conditions for reconditioning. The column oven temperature was kept at $40{ }^{\circ} \mathrm{C}$. The injection volume was $10 \mu \mathrm{L}$.

\section{Animals and experimental treatment}

Male Sprague-Dawley rats ( 250 g, 2 months old) were obtained from Nara Bio Co. (Seoul, Korea). Rats were housed separately (four animals per cage) under a temperaturecontrolled room $\left(25 \pm 2{ }^{\circ} \mathrm{C}\right.$, humidity $55 \%, 12 \mathrm{~h}$ light/dark cycle) and provided free access to water and food. After 1 week, the rats were randomly assigned to one of four experimental groups $(n=10)$ for an additional 28 days. These groups were the control group, the HFD group, the low-dose OFP-EA-extract $(100 \mathrm{mg} / \mathrm{kg} / \mathrm{day}$ p.o.) treated group and the high-dose OFP-EAextract (300 mg/kg/day p.o.) treated group. The control group was fed with standard normal rat chow with protein $(\sim 14 \%)$, fat $(\sim 10 \%)$ and carbohydrate $(\sim 76 \%)$, whereas the other groups were fed with HFD, which is a standard rat chow supplemented with $1 \%$ cholic acid, $2 \%$ pure cholesterol and $5.5 \%$ oil. The rats were administered with distilled water or OFP-EA extract (100 and $300 \mathrm{mg} / \mathrm{kg}$ ) by oral gavage (20 $\mathrm{mL} / \mathrm{kg}$ ) once every morning for 28 days. At the end of the experimental period, the rats were fasted overnight and sacrificed by cervical dislocation. Blood samples and liver tissues were then collected for further analysis. All experiments were performed under the guidelines of Principles of Laboratory Animal Care (NIH publication No. 85-23, revised 1985) and our Institutional Animal Care and Use Committee, Konkuk University.

\section{Analysis of lipid levels in blood samples}

To obtain the baseline lipid profiles in plasma, fasted blood samples $(2 \mathrm{~mL})$ were collected from the right orbital sinus of rats anesthetized with diethyl ether through a small heparinized capillary tube before the experiment. After the 28-day treatment period, rats were sacrificed by cervical dislocation. Fasted blood samples (2 $\mathrm{mL}$ ) were collected in chilled heparinized centrifuge tubes by cardiac puncture with heparinized syringes. The blood samples were centrifuged $(1500 \times \mathrm{g})$ at $4{ }^{\circ} \mathrm{C}$ for $15 \mathrm{~min}$. The collected plasma samples were stored at $-80^{\circ} \mathrm{C}$ until further analysis. Plasma levels of TC, TG, LDL and HDL were measured by using the Asan Biotech (Seoul, Korea).

\section{Gross appearance and lipid content of liver}

At the end of the study, the whole liver was harvested from rats and rinsed in saline (three to four times) to remove as much blood as possible. The gross appearance of livers of individual rat was photographed for comparison. The sample of liver $(\sim 2 \mathrm{~g})$ was dissected and homogenized with $2: 1$ chloroform-methanol mixture $(\mathrm{v} / \mathrm{v})$ to a final dilution of 1:20 w/v using Ultra-turrax T-25 homogenizer. After filtration, $10 \mathrm{~mL}$ of filtrate was added to $2 \mathrm{~mL}$ water, and the mixture was centrifuged at $900 \mathrm{~g}$ for $20 \mathrm{~min}$. The lower phase was dried, and its weight was measured. Liver lipid content was expressed as weight of lipid per gram of liver.

\section{Western blot analysis}

Hepatic HMGR, ACAT, CYP7A1 and PPAR- $\alpha$ protein levels were quantified using western blotting procedures. Liver tissues from various experimental groups were isolated $\left(\begin{array}{ll}\sim \mathrm{g}\end{array}\right)$, weighed and kept at $-80^{\circ} \mathrm{C}$ immediately until homogenization procedures. Each frozen sample was homogenized on ice in lysis buffer consisting of $50 \mathrm{mM}$ Tris- $\mathrm{HCl}(\mathrm{pH} 8.0), 150 \mathrm{mM} \mathrm{NaCl}, 1 \%$ Triton X-100, $0.5 \%$ sodium deoxycholate, $0.1 \%$ sodium dodecyl sulfate (SDS), $1 \mathrm{mM}$ EDTA (Sigma), 1\% protease inhibitor cocktail, and $1 \%$ phosphatase inhibitor cocktail (Roche). After centrifugation, the protein concentration of the supernatant was assayed using a Bio-Rad DC Protein Assay kit. Lysate samples (containing 20 $\mu \mathrm{g}$ for HMGR and ACAT; $40 \mu \mathrm{g}$ for CYP7A1 and PPAR- $\alpha$ ) of protein were fractionated by SDS-10 $\%$ polyacrylamide gel electrophoresis and then electroblotted onto polyvinylidene difluoride membranes (Millipore, Bedford, MA, USA). The membranes were probed with primary antibodies to anti-HMGR, anti-ACAT, anti-CYP7A1 (1:1000), anti- $\beta$-actin (1:2000), and anti-PPAR (1:500). The blots were visualized by a PowerOpti-ECL (Animal Genetics Inc, Tallahassee, FL, USA) detection system according to the manufacturer's procedure and the antibody-specific bands were scanned by a Luminescent Analyzer, LAS-3000 and quantified using Fuji Multigage software V3.1 (Fuji, Tokyo).

\section{Statistical analysis}

Data were expressed as means \pm SEM, and $n$ denotes the number of replications for each data point. After validation of each parameter for homogeneity of variance, the differences between groups were assessed by one-way ANOVA using Statistical Package for the Social Sciences for Windows (version 12; SPSS Inc., Chicago, IL, USA). Post hoc testing was 
performed for intergroup comparisons using the least significance difference test; $p$ values less than 0.05 were considered statistically significant.

\section{RESULTS}

\section{Identification of active constituent, hydroxytyrosol from OFP-EA extract}

The HPLC finger printing analysis of OFP-EA extract was shown in Fig. 1. Earlier studies indicated that olive fruit contains polyphenolic compound including oleuropein, hydroxytyrosol, tyrosol, 4- hydroxyphenyl acetic acid, protocatechuic acid, caffeic acid and p-coumaric acid [14,15]. Espeacially, hydroxytyrosol shows a broad spectrum of beneficial effects such as cancer chemoprevention, cardioprotection, skin photoprotection and anti-inflammatory activity [16]. Therefore, the major peaks obtained in our HPLC fingerprint analysis were compared with standard hydroxytyrosol. Hydroxytyrosol was identified by HPLC/UV analysis using retention times and absorption spectra in the $200-380$ $\mathrm{nm}$ range (Fig 1B).

\section{Plasma cholesterol and lipoprotein profiles}

The plasma lipid profiles (TC, TG, HDL and LDL) from various treatment groups of rats are summarized in Table 1. Elevated levels of TC and LDL with a decreased HDL were observed in rats fed with HFD. Treatment with OFP-EAextract $(100$ and $300 \mathrm{mg} / \mathrm{kg})$ significantly suppressed the increased plasma TC and LDL induced by the HFD. OFP-EA-extract (300 $\mathrm{mg} / \mathrm{kg} /$ day) significantly reduced the plasma total cholesterol $(4.81 \pm 0.31 \mathrm{mM} / \mathrm{L}, p<0.01)$ and LDL $(6.92 \pm 0.70 \mathrm{mM} / \mathrm{L}, p<0.05)$, as compared with those in the HFD group (total cholesterol, $5.67 \pm 0.32 \mathrm{mM} / \mathrm{L} ; \quad$ LDL, $9.91 \pm 1.25 \mathrm{mM} / \mathrm{L})$. However, both the plasma total cholesterol and LDL contents did not return to normal level in the OFP-EA-extract (300 mg/kg/day) group. Animals treated with OFP-EA extract with HFD showed no significant change on the level of triglycerides. The level of HDL in the HFD was significantly lowered than that in the control group $(p<0.001)$. However treatment with OFP-EA-extract (300 $\mathrm{mg} / \mathrm{kg} /$ day) group (but not in the $100 \mathrm{mg} / \mathrm{kg} /$ day group), significantly enhanced the decreased plasma HDL as compared with the HFD group $(p<0.01)$.
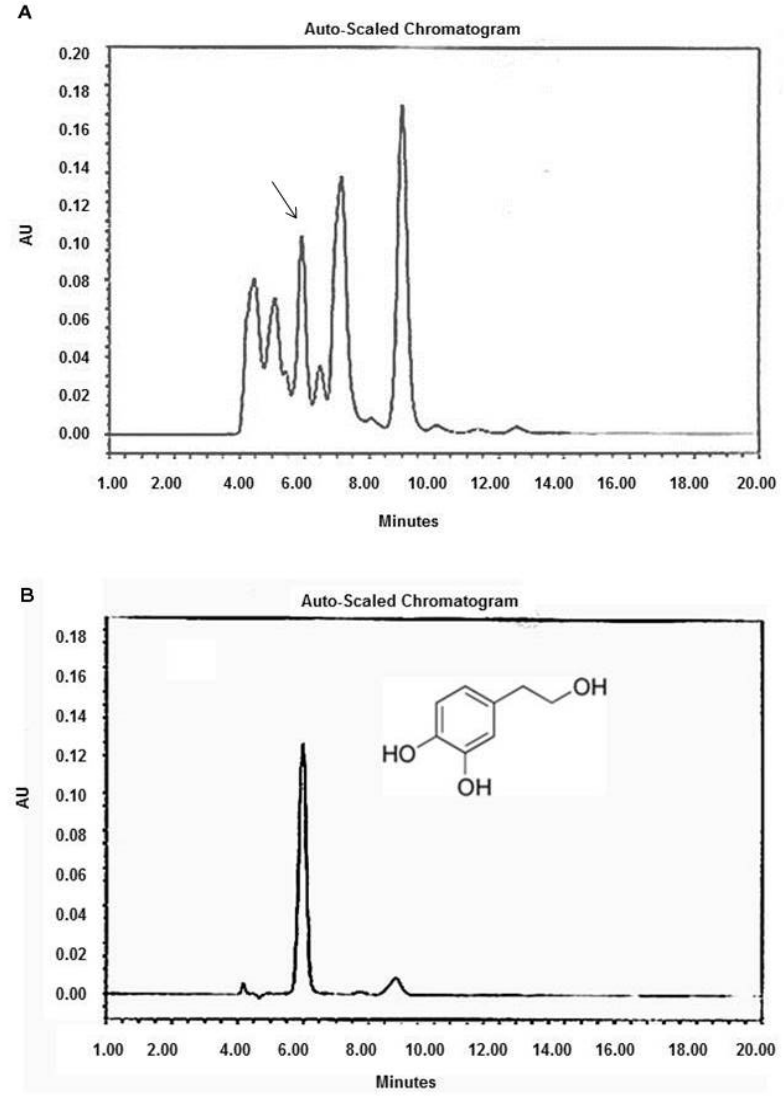

Fig 1: HPLC chromatogram of OFP-EA extract. $A=$ identification of major peaks in OFP-EA extract. The arrow shown is the target compound, hydroxytyrosol; $\mathrm{B}=$ comparison with respective standard and structure of hydroxytyrosol. OFP-EA = Olea europea-ethyl acetate.

Further, to evaluate the effect of plasma lipid profiles on the progression of atherosclerosis and

Table 1: Plasma lipid levels of rats after administering 100 and $300 \mathrm{mg} / \mathrm{kg}$ doses, respectively

\begin{tabular}{|c|c|c|c|c|c|}
\hline \multicolumn{2}{|c|}{ Animal groups } & Control & HFD & OFPEA-100 & OFP-EA-300 \\
\hline \multirow[t]{2}{*}{$\mathrm{TC}(\mathrm{mM})$} & Before & $1.87 \pm 0.13$ & $2.23 \pm 0.07$ & $2.46 \pm 0.10$ & $2.27 \pm 0.10$ \\
\hline & After & $1.73 \pm 0.07$ & $5.67 \pm 0.32^{\# \# \#}$ & $5.45 \pm 0.27^{\# \# \#}$ & $4.81 \pm 0.31^{\# \# \#, *}$ \\
\hline \multirow[t]{2}{*}{ TG (mM) } & Before & $0.70 \pm 0.01$ & $0.71 \pm 0.01$ & $0.71 \pm 0.01$ & $0.70 \pm 0.01$ \\
\hline & After & $0.70 \pm 0.01$ & $0.68 \pm 0.01$ & $0.65 \pm 0.01$ & $0.61 \pm 0.01$ \\
\hline \multirow[t]{2}{*}{ LDL (mM) } & Before & $0.68 \pm 0.03$ & $0.81 \pm 0.11$ & $0.82 \pm 0.08$ & $0.92 \pm 0.07$ \\
\hline & After & $0.40 \pm 0.04$ & $9.91 \pm 1.25^{\# \# \#}$ & $8.74 \pm 0.60^{\# \# \#}$ & $6.92 \pm 0.70^{\# \# \#, *}$ \\
\hline \multirow[t]{2}{*}{$\mathrm{HDL}(\mathrm{mM})$} & Before & $0.94 \pm 0.07$ & $1.05 \pm 0.02$ & $1.10 \pm 0.05$ & $1.08 \pm 0.05$ \\
\hline & After & $0.91 \pm 0.05$ & 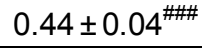 & $0.59 \pm 0.05^{\# \# \#}$ & $0.65 \pm 0.07^{\# \#, * *}$ \\
\hline
\end{tabular}



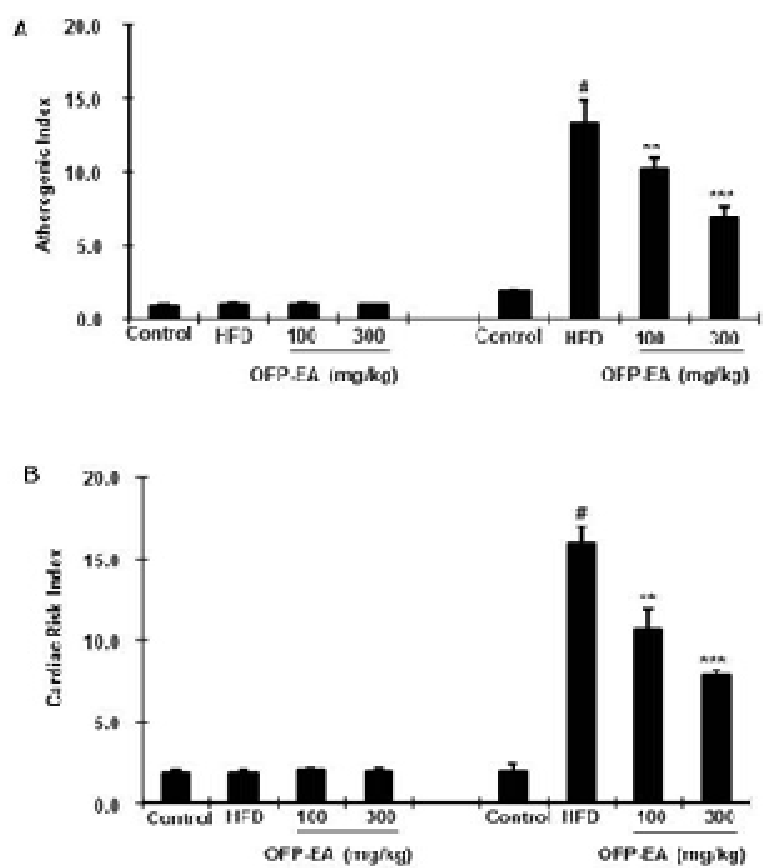

Fig 2: Effect of OFP-EA extract on atherogenic index and cardiac risk factor in HFD fed rats. The atherogenic index $(A)$ and cardiac risk factor $(B)$ of animals from the control, HFD, OFP-EA-extract (100 $\mathrm{mg} / \mathrm{kg} /$ day $)$ and OFP-EA-extract (300 $\mathrm{mg} / \mathrm{kg} /$ day $)$ groups were shown. Data are expressed as mean \pm SEM, $\mathrm{n}=8-10$. ${ }^{*} p<0.001$ compared with the control group. ${ }^{* *} p<0.01$ and ${ }^{* * *} p<0.001$ when compared with the HFD fed group (HFD: high fat diet; OFP-EA: Olea europea-ethyl acetate)

heart disease, the atherogenic index [(total serum cholesterol-HDL)/HDL] and the cardiac risk factor (total serum cholesterol/HDL) were measured, respectively (Fig 2). It was found that both the atherogenic index and the cardiac risk factor were elevated markedly in the HFD group. However, there was significant decrease in the atherogenic index $(p<0.01)$ and the cardiac risk factor $(p<0.01)$ in animals treated with OFP-EAextract (300 mg/kg/day) compared with the HFD group (Fig 2A and 2B). It suggested that OFP-EA treated at high doses $(300 \mathrm{mg} / \mathrm{kg})$ showed atheroscleroprotective and cardioprotective potential in the current experimental setting.

\section{Gross appearance and lipid content of liver}

The gross appearances of the rat livers from different treatment groups are depicted in the Fig 2B. The liver from the control group showed a relatively dark-red colour, whereas the liver from the HFD group showed a yellowish colour. In both OFP-EA-extract treated groups (100 and $300 \mathrm{mg} / \mathrm{kg}$ dose), the isolated livers have a relatively lesser yellowish colour as compared with that from the HFD group. To evaluate the lipid content in the livers from different treatment groups, total lipid in the liver was isolated and compared. There was significant increase $(p<0.05)$ in liver lipid content in HFD group as compared with that of the control group. Interestingly, supplementing the animals with 100 and $300 \mathrm{mg} / \mathrm{kg} /$ day of OFP-EA-extract could dose-dependently reduce the increased lipid content in livers of hypercholesterolemic rats (Fig 2B).

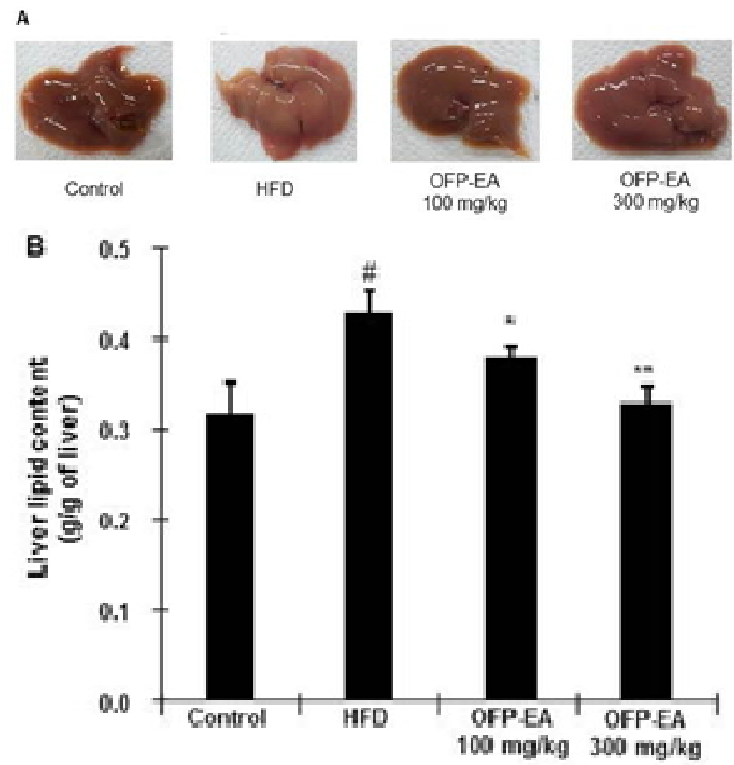

Fig 3: Effect of OFP-EA extract on gross appearance and lipid content of liver (A) The gross appearance of the entire liver obtained from rats from the control, HFD, OFP-EA-extract (100 mg/kg/day) and OFP-EAextract(300 mg/kg/day) groups. (B) Total lipid content per gram of liver isolated from various indicated groups. Data are expressed as means \pm SEM, $n=8-$ 10. ${ }^{\#} p<0.001$ compared with the control group. * $p<0.05$ and ${ }^{* *} p<0.01$ when compared with the HFD fed group. HFD: high fat diet; OFP-EA: Olea europeaethyl acetate. (HFD: high fat diet; OFP-EA: Olea europea-ethyl acetate)

\section{Western blot results}

Molecular mechanisms of OFP-EA extract in the cholesterol metabolic pathway in liver were further studied by evaluating the changes in the protein expressional levels of hydroxymethylglutaryl-CoA reductase (HMGR), acyl-. CoA:cholesterol acyltransferase (ACAT), cytochrome P450 7A1 (CYP7A1) and peroxisome proliferation-activated receptor alpha (PPAR- $\alpha$ ) (Fig 4A). Quantification data revealed that the protein expression levels of HMGR in the livers from all the groups fed with HFD were markedly lower than that of the control group (Fig 4B). Supplementing the hypercholesterolemic rats with OFP-EA extract at indicated doses (100 and $300 \mathrm{mg} / \mathrm{kg}$ ) showed no apparent change in the protein expression levels of HMGR. 
However, the protein expressions of ACAT, CYP7A1 and PPAR- $\alpha$ in liver, were significantly increased in the HFD group as compared with that in the control group $(p<0.001$, Fig. 4B). Treatment with OFP-EA extract in hypercholesterolemic rats showed no apparent change in the protein expression level of ACAT. Interestingly, hypercholesterolemic rats treated with OFP-EA extract showed dose-dependent down-regulation in the protein expression of CYP7A1 and up-regulation in the protein expression of PPAR- $\alpha$ as compared with the HFD fed rats.
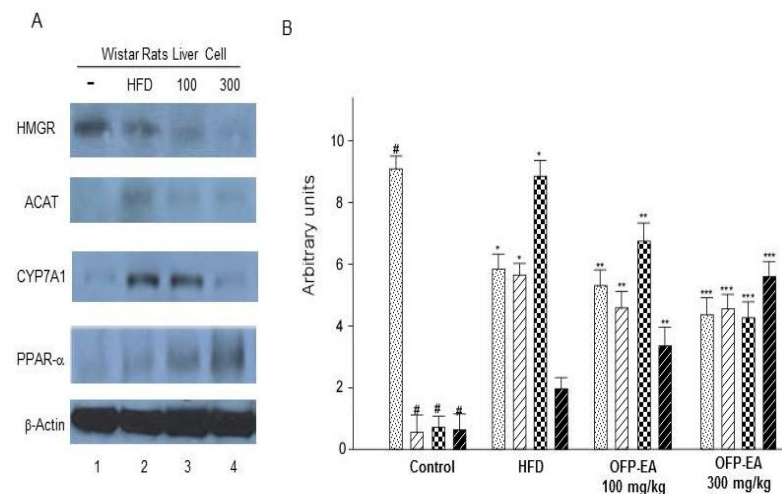

Fig 4: Effect of OFP-EA extract on the protein expressions of HMGR, ACAT, CYP7A1 and PPAR- $\alpha$ in HFD fed rats. A: The protein expression of HMGR, ACAT, CYP7A1 and PPAR- $\alpha$ from isolated livers were evaluated by Western blotting. The expression level of each gene was normalized to that of the $\beta$-actin protein. B: The quantification data of each protein expression (HMGR, ACAT, CYP7A1 and PPAR- $\alpha$ ) from the control, HFD, OFP-EA-extract(100 $\mathrm{mg} / \mathrm{kg} /$ day) and OFP-EA-extract(300 $\mathrm{mg} / \mathrm{kg} /$ day) groups were shown. Data are expressed as means \pm S.E.M, $n=8-10 .{ }^{\#} p<0.001$ compared with the control group. ${ }^{* *} p<0.01$ and ${ }^{* * *} p<0.001$ when compared with the HFD fed group. HFD: high fat diet; OFP-EA: Olea europea-ethyl acetate, HMGR: Hydroxymethylglutaryl-CoA reductase, ACAT: AcylCoA:cholesterol acyltransferase, CYP7A1: Cytochrome P450 7A1, PPAR-a: Peroxisome proliferation-activated receptor alpha. $0 \mathrm{HMGR} / \beta$ actin, $\square$ ACAT/ $\beta$-actin, $\mathrm{CYP7A} 1 / \beta$-actin, 2 PPAR- $\alpha /$ $\beta$-actin.

\section{DISCUSSION}

Diet plays an important role in the regulation of cholesterol homeostasis. In recent decades, functional food and natural dietary substances from natural herbs possessing hypolipidemic and hypocholesterolemic properties have been extensively studied to determine their role in preventing CVD $[17,18]$. In this study, the effects of OFP-EA-extract on the plasma lipid profiles of HFD-fed rats were evaluated. Levels of TC and LDL were elevated, whereas HDL was lowered as a result of consuming HFD for 28 days. Our results are consistent with previous findings with similar experimental settings as there was no significant difference on plasma TG among various groups $[18,19]$. The supplementation of a high dose of OFP-EA extract $(300 \mathrm{mg} / \mathrm{kg})$ significantly altered the abnormal plasma lipid level induced by HFD. Reductions in the TC and LDL, but alleviation of HDL, were observed at the end of the 28-day treatment period. These results are in agreement with those reported in earlier studies with chlorogenic acid treatment in hypercholesterolemic animals [20,21]. Clinical and epidemiological studies have proved that people with elevated LDL have an increased risk for CVD [22]. In addition, HDL may be protective by reversing cholesterol transport, by inhibiting the oxidation of LDL and by neutralizing the atherogenic effects of oxidized LDL [23]. The supplementation of OFP-EA-extract was effective in lowering LDL and raising HDL contents in hypercholesterolemic rats.

In fact, the risk of CVD can also be expressed by atherogenic index and cardiac risk factor. In this study, the elevated atherogenic index and cardiac risk factor induced by HFD was suppressed significantly by OFP-EA extract $(p<$ 0.01 at $100 \mathrm{mg} / \mathrm{kg}$ and $p<0.001$ at $300 \mathrm{mg} / \mathrm{kg}$ dose respectively). This suggested that OFP-EA extract consumption could provide atheroscleroprotective effect to the body. Although OFP-EA-extract treatment did not completely normalized the two indicators in the HFD group, the findings were still strong enough to demonstrate that OFP-EA-extract possessed atheroscleroprotective and cardioprotective properties by lowering plasma cholesterol, restoring the $\mathrm{HDL}$ and improving both atherogenic index and cardiac risk factor. It was well documented that hypercholesterolemic diet could affect the functions of liver substantially because of the high-cholesterol condition that causes an excessive production of reactive oxygen species (ROS), which in turn could initiate lipid peroxidation, damage liver functions, and affect the cardiovascular system [24-26].

Non-alcoholic fatty liver disease (NAFLD) is one of the most common chronic liver disorders, having a broad spectrum ranging from simple steatosis to cirrhosis [27-29]. OFP-EA-extract, as shown in the current study, exerted beneficial effects in reducing the enlargement of liver as indicated by a significant decrease in liver lipid content in hypercholesterolemic rats.. Therefore, the results suggested that OFP-EA-extract may have hepatoprotective effects (in terms of reducing the risk of developing the condition of hepatomegaly and/or fatty liver) by preventing the liver from having depositions of fat and 
cholesterol. The liver is the most important organ for the intermediary metabolism of lipids, and also manufactures cholesterol for normal body functions. Therefore, preventing and treating NAFLD are relevant to health promotion. Therefore, we studied the effects of OFP-EAextract treatment on the cholesterol metabolismrelated gene (HMGR, ACAT and CYP7A1) expressions in hypercholesterolemic rats by using real-time PCR analysis. HMGR are enzymes responsible for cholesterol synthesis, whereas ACAT and CYP7A1 are enzymes responsible for catalyzing cholesterol esterification and bile acid synthesis in expending of cholesterol [30].

It is believed that HMGR are regulated through negative feedback of cholesterol [30]. Low cholesterol level would trigger HMGR and CYP51 transcriptions so as to enhance cholesterol synthesis. In this study, the reduced transcriptions of these two enzymes in hypercholesterolemic rats may be mediated by HFD. From our results, the suppressed protein expressions of HMGR were observed in the HFD fed rats and OFP-EA-extract treatments. There was no significant difference of HMGR and CYP51 protein levels among all the groups fed with HFD and OFP-EA treated animals. Hence, the hypocholesterolemic effect exerted by OFP. EA-extract may not be related to the inhibition of cholesterol synthesis.

Excess cholesterol is often stored as cholesteryl esters by esterification, and this process is catalyzed by the microsomal enzyme, ACAT [31]. ACAT in liver is also involved in the synthesis of very low density lipoprotein [32]. In the present study, the protein expression level of ACAT showed a significant increase in all rats with a high-cholesterol diet as compared with the control group. OFP-EA-extract treatment could not alter the increased mRNA expression of ACAT in hypercholesterolemic rats, suggesting that ACAT was not a target for OFP-EA-extract.

CYP7A1 is a rate-determining enzyme in the biosynthetic pathway of bile acids by expending cholesterol in liver and is the major cholesterol elimination mechanism [31]. The expression level of CYP7A1 was shown to be up-regulated by high plasma cholesterol, suggesting that the synthesis of bile acid is positively dependent on plasma cholesterol level [19]. In this study, the increased CYP7A1 protein expression in hypercholesterolemic rats was attenuated dose dependently by OFP-EA-extract treatment. The down-regulation of CYP7A1 was most likely an outcome instead of a cause of reduced plasma cholesterol levels in OFP-EA-extract treated animals as plasma cholesterol and CYP7A1 expression level are positively correlated.

PPARs are members of the nuclear receptor superfamily of ligand-binding transcription factors that have important effects on lipid homeostasis through the regulation of the expression of genes involved in lipid metabolism. PPAR- $\alpha$ is predominantly expressed in the liver and regulates the transcription of genes involved in hepatic fatty acid uptake and oxidation [33,34]. , and agonists of PPAR- $\alpha$ are shown to be antihyperlipidemic agents [35]. PPAR- $\alpha$ mRNA expression was positively correlated with dietary fat intake in rats because an increased supply of fatty acids, endogenous ligand for PPAR-a receptor, might contribute to an increased PPAR$\alpha$ signaling and up-regulated PPAR- $\alpha$ mRNA expression [36]. In the current experimental settings, hypercholesterolemic rats showed an up-regulation of PPAR- $\alpha$ mRNA expression. This might be due to the extra $5.5 \%$ oil in the diet, which provided more fatty acids to stimulate PPAR- $\alpha$ receptors in the liver. The further upregulation of PPAR- $\alpha$ mRNA expression in animals consuming OFP-EA-extract could explain the hypocholesterolemic effect of OFP. EA-extract.

One of the most extensively studied phenolic compounds in olives is hydroxytyrosol, an odiphenol with marked antioxidant activity. Hydroxytyrosol shows a broad spectrum of beneficial effects such as cancer chemoprevention, cardioprotection, skin photoprotection and anti-inflammatory activity [14-16]. Experimental studies also showed that purified hydroxytyrosol from olive mill waste possess antioxidant effects in alloxan-induced diabetic rats by increasing endogenous antioxidant enzymes and decreasing plasma thiobarbituric acid reactive substances [37]. These biological properties are based on scavenging of reactive oxygen species by hydroxytyrosol, being more active than antioxidant vitamins and synthetic antioxidants [37]. In our present study, HPLC fingerprinting analysis of OFP-EA extract revealed hydroxytyrosol as one of the major active constituent. Therefore the potent antioxidant polyphenolic compounds such as hydroxytyrosol present in OFP-EA extract might also help in synergizing the hypocholesterolemic and hepatoprotective effects.

\section{CONCLUSION}

The present results indicate for the first time that supplementation of OFP-EA-extract has significant hypocholesterolemic and hepatoprotective effects. Increase in fatty acids unitization 
in liver of rats fed with high-fat diet and the upregulation of PPAR- $\alpha$ expression might probably be the mechanism. Further the anti-oxidant compounds present in OFP-EA extract might also synergistically act to enhance the hypocholesterolemic and hepatoprotective activities. Therefore, dietary consumption of OFP-EA could have beneficial impact on cardiovascular and liver disorders.

\section{ACKNOWLEDGEMENT}

This research was supported by a grant from the Regional Strategic Planning Technology Development Program funded by the Ministry of Knowledge Economy of the Korean government and by a grant from Macrocare Tech., Ltd. The authors thank Chungbuk Technopark for excellent administrative assistance. Kim MS and Koppula S contributed equally to this work.

\section{COMPETING INTEREST}

The authors declare that there are no conflicts of interest.

\section{REFERENCES}

1. Tripoli E, Giammanco M, Tabacchi G, Di Majo D. The phenolic compounds of olive oil: structure, biological activity and beneficial effects on human health. Nutr Res Rev 2005; 18: 98-112.

2. Boskou G, Salta FN, Chrysostomou S, Mylona A Antioxidant capacity and phenolic profile of table olives from the Greek market. Food Chem 2006; 94: 558-564.

3. Arbones-Mainar JM, Ross K, Rucklidge GJ, Reid M. Extra virgin olive oils increase hepatic fat accumulation and hepatic antioxidant protein levels in APOE(-/-) mice. J Proteome Res 2007; 6: 40414054.

4. Menendez JA, Vazquez-Martin A, Garcia-Villalba $R$, Carrasco-Pancorbo A. Anti-HER2 (erbB-2) oncogene effects of phenolic compounds directly isolated from commercial extra-virgin olive oil (EVOO). BMC Cancer 2008; 8: 377.

5. Visioli $F$, Galli $C$. The effect of minor constituents of olive oil on cardiovascular disease: new findings. Nutr Rev 1998; 56: 142-147.

6. Kim MS, Koppula S, Jung SH, Kim JY, Lee HR, Lee SR, Park YD, Lee KA, Park TK, Kang H. Olea europaea Linn (Oleaceae) fruit pulp extract exhibits potent antioxidant activity and attenuates neuroinflammatory responses in lipopolysaccharide- stimulated microglial cells. Trop J Pharm Res 2013; 12: 357-362.

7. Fabiani $R$, Rosignoli $P$, De Bartolomeo $A$, Fuccelli $R$. Oxidative DNA damage is prevented by extracts of olive oil, hydroxytyrosol, and other olive phenolic compounds in human blood mononuclear cells and HL60 cells. J Nutr 2008; 138: 1411-1416.

8. Fernandez-Bolanos JG, Lopez O, Fernandez-Bolanos J, Rodriguez-Gutierrez G. Hydroxytyrosol and derivatives: isolation, synthesis, and biological properties. Curr Org Chem 2008; 12: 442-463.

9. Toth PP. Atherosclerosis: The Underlying Disease. J Fam Pract 2009; 58: S19-S25.
10. Suanarunsawat T, Devakul Na, Ayutthaya W, Songsak T, Thirawarapan S, Poungshompoo S. Antioxidant Activity and Lipid-Lowering Effect of Essential Oils Extracted from Ocimumsanctum L. Leaves in Rats Fedwith a High Cholesterol Diet. J Clin Biochem Nutr 2010; 46: 52-59.

11. Festi $D$, Colecchia $A$, Sacco $T$, Bondi $M$, Roda $E$, Marchesini G. Hepatic steatosis in obese patients: clinical aspectsand prognostic significance. Obes Rev 2004; 5: 27-42.

12. TeSligte $K$, Bourass $I$, Sels JP, Driessen $A$, Stockbrugger R.W, Koek GH. Non-alcoholic steatohepatitis: review of a growing medical problem. Eur J Intern Med 2004; 15:10-21.

13. Kwok CY, Wong CNY, Yau MYC. Consumption of dried fruit of Crataeguspinnatifida (hawthorn) suppresses highcholesterol diet-induced hypercholesterolemia in rats. J Functional Foods 2010; 2: 179-186.

14. Cook NC, Samman S. Flavonoids-chemistry, metabolism,cardioprotective effects, and dietary sources. Nutr Biochem 1996; 7: 66- 76.

15. Caponio F, Alloggio V, Gomes T. Phenolic Compounds of Virgin Olive Oil: Influence of Paste Preparation Techniques. Food Chem 1999; 64: 203-209.

16. Jose FB, Oscar L, Juan FB and Guillermo RG. Hydroxytyrosol and Derivatives: Isolation, Synthesis, and Biological Properties. Cur Org Chem 2008; 12: 442-463.

17. Li Q, Wu JH, Guo DJ, Cheng HL, Chen SL, Chan SW. Suppression of diet-induced hypercholesterolemia by scutellarinin rats. Planta Med 2009; 75: 12031208.

18. Yan LP, Chan SW, Chan AS, Chen SL, Ma XJ, Xu HX. Puerarin decreases serum total cholesterol and enhances thoracic aortaendothelial nitric oxide synthase expression in diet-induced hypercholesterolemic rats. Life Sci 2006; 79: 324330.

19. Yiu WF, Kwan PL, Wong CY. Attenuation of fatty liver andprevention of hypercholesterolemia by extract of Curcuma longathrough regulating the expression of CYP7A1, LDL-receptor, HO-1, and HMG-CoA reductase. J Food Sci 2011; 76: H80-H89.

20. Karthikesan K, Pari L, Menon VP. Antihyperlipidemic effect of chlorogenic acid and tetrahydro curcumin in rats subjectedto diabetogenic agents. Chem Biol Interact 2010; 188: 643-650.

21. Rodriguez de Sotillo DV, Hadley M. Chlorogenic acid modifiesplasma and liver concentrations of: cholesterol, triacylglycerol,and minerals in (fa/fa) Zucker rats. J Nutr Biochem 2002; 13: 717-726.

22. Keevil JG, Cullen MW, Gangnon R, McBride PE, Stein $\mathrm{JH}$. Implications of cardiac risk and low-density lipoprotein cholesteroldistributions in the united states for the diagnosis and treatment of dyslipidemia-data from National Health and Nutrition ExaminationSurvey 1999 to 2002. Circulation 2007; 115: 1363-1370.

23. Parthasarathy S, Barnett J, Fong LG. High-DensityLipoprotein Inhibits the Oxidative Modification of Low-Density-Lipoprotein. Biochem Biophys Acta 1990; 1044: 275-283.

24. Kojda G, Harrison D. Interactions between $N O$ and reactive oxygen species: pathophysiological importance in atherosclerosis, hypertension, diabetes and heart failure. Cardiovascular Research 1999; 43: 562-571.

25. Lum $H$, Roebuck KA. Oxidant stress and endothelial cell dysfunction. American Journal of Physiology 2001; 280: C719-C741.

26. Martinet W, Knaapen MWM, De Meyer GRY, Herman AG, Kockx MM. Oxidative DNA damage and repair in experimental atherosclerosis are reversed by dietary lipid lowering. Circulation Research 2001; 88: 733-739. 
27. Ludwig J, Viggiano TR, McGill DB, Oh BJ. Nonalcoholic steatohepatitis: Mayo Clinic experiences with a hitherto unnamed disease. Mayo Clin Proc 1980; 55: 434-8.

28. Farrell GC, Larter CZ. Nonalcoholic fatty liver disease: from steatosis to cirrhosis. Hepatology 2006; 43 : S99-112.

29. Ludwig J, McGill DB, Lindor KD. Review: nonalcoholic steatohepatitis. J Gastroenterol Hepatol 1997; 12: 398-403.

30. Dietschy JM. Theoretical considerations of what regulates low-density-lipoprotein and high-densitylipoprotein cholesterol. Am J Clin Nutr 1997; 65: 1581S-1589S

31. Chiang JY. Regulation of bile acid synthesis. Front Biosci 1998; 3: d176-d193.

32. Azuma Y, Kawasaki T, Ikemoto K. Cholesterol lowering effects of NTE-122, a novel acylCoA:cholesterolacyltransferase (ACAT) inhibitor, on cholesterol diet-fed ratsand rabbits. Jpn J Pharmacol 1998; 78: 355-364.
33. Escher $P$, Wahli W. Peroxisome proliferator-activated receptors:insight into multiple cellular functions. Mutat Res-Fund Mol 2000; M448: 121-138.

34. Leone TC, Weinheimer CJ, Kelly DP. A critical role for theperoxisome proliferator-activated receptor alpha (PPAR alpha) in the cellular fasting response: The PPAR alpha-null mouseas a model of fatty acid oxidation disorders. Pro Natl Acad Sci USA 1999; 96: 7473-7478.

35. Avogaro A, Miola M, Favaro A. Gemfibrozil improves insulin sensitivity and flow-mediated vasodilatation in type 2 diabetic patients. Eur J Clin Invest 2001; 31: 603-609.

36. Hsu SC, Huang CJ. Changes in liver PPAR alpha mRNAexpression in response to two levels of highsafflower-oil dietscorrelate with changes in adiposity and serum leptin in ratsand mice. $J$ Nutr Biochem 2007; 18: 86-96.

37. Hamden $K$, Allouche $N$, Damak $M$, Elfeki $A$. Hypoglycemic and antioxidant effects of phenolic extracts and purified hydroxytyrosol from olive mill waste in vitro and in rats. Chem Biol Interact 2009; 180: 421-432. 